\title{
Ações educativas realizadas com profissionais da atenção primária à saúde na região da Amazônia Ocidental Brasileira
}

Educational actions carried out with primary health care professionals in the region of the Brazilian Western Amazon

Actividades educativas con los profesionales de atención primaria de salud en la región occidental de la Amazonia brasileña

Lúcia Dias da Silva Guerra ${ }^{1}$

\section{Resumo}

Ações educativas em alimentação e nutrição, de acordo com as políticas públicas brasileiras, são um caminho estratégico para a realização da Segurança Alimentar e Nutricional (SAN) para maximizar a lógica da Promoção da Saúde e efetivar o Direito Humano à Alimentação Adequada. Este relato de experiência tem o objetivo de apresentar as ações educativas realizadas com Agentes Comunitários de Saúde (ACS) e nutricionistas da equipe Estratégia Saúde da Família da Atenção Primária, da região da Amazônia Ocidental Brasileira. Para isso, utilizou-se como fundamentação metodológica a investigação pedagógica através de uma a abordagem qualitativa. Como suporte interpretativo, usou-se a compreensão da alimentação como um ato biosociocultural, de SAN e de Educação Alimentar e Nutricional (EAN). As ações educativas foram realizadas no município de Acrelândia, Acre, com 45 ACS e duas nutricionistas; e desenvolvidas em quatro etapas: diagnóstico, planejamento, execução e avaliação. Nesta experiência de pesquisa foram produzidas quatro ações, por meio de oficinas educativas e oficinas culinárias. Pelo identificado, parece fundamental repensar as práticas de EAN considerando a equipe multiprofissional, bem como o usuário e o território, como uma proposta para se realizar o cuidado nutricional na perspectiva da Atenção Primária à Saúde.

Descritores: Atenção primária à saúde. Educação alimentar e nutricional. Hábitos alimentares. Promoção da saúde. Ecossistema amazônico.

\footnotetext{
Abstract

According to the Brazilian public policy, food and nutrition education activities are presented as strategies to the accomplishment of Food and Nutritional Security (SAN), maximization of the Health Promotion and implementation the Human Right to Adequate Food. This experience report aims to present the educational activities with Community Health Agents (ACS) and dietitians in Family Health Strategy team in Primary Care, at the western Brazilian Amazon region. In this way, the educational research through a qualitative approach was used as methodological basis. For interpretative support, feed was understood as a biosociocultural, SAN and Food and Nutrition Education (EAN) act. Educational activities happened in the municipality of Acrelândia, Acre, with 45 ACS and two dietitians. They were developed in four stages: diagnosis, planning, implementation and evaluation. In this research experience were produced four interventions through educational workshops and culinary workshops. The results suggest a fundamental rethink

${ }^{1}$ Doutoranda no Programa de Pós-Graduação de Nutrição em Saúde Pública, Faculdade Saúde Pública, Universidade de São Paulo. Nutricionista, Mestre em Saúde Coletiva. Endereço: Universidade de São Paulo. Faculdade de Saúde Pública. Centro de Estudos e Pesquisas de Direito Sanitário - CEPEDISA. Av. Dr. Arnaldo, no 715 . São Paulo - SP, 01246 -904. email: ludsguerra@ gmail.com.
} 
ISSN 2179-6750

EAN practices considering the multidisciplinary team, as well as the user and the territory as a proposal to execute the nutritional care in the context of Primary Health Care.

Key-words: Primary health care. Food and nutrition education. Food habits. Health promotion. Amazonian ecosystem.

\section{Resumen}

Acciones educativas en alimentación y nutrición, de acuerdo con las políticas públicas brasileñas, se muestran como camino estratégico para: el alcance de la Seguridad Alimentaria y Nutricional (SAN), la maximización de la lógica de la Promoción de la Salud y la realización del derecho humano a la alimentación adecuada. Este relato de experiencia tiene como objetivo presentar las acciones educativas con los Agentes Comunitarios de Salud (ACS) y nutricionistas del equipo "Estrategia de Salud de la familia", de la región amazónica occidental del Brasil. Para ello, se empleó como fundamento metodológico la investigación pedagógica a través de un enfoque cualitativo. Como soporte interpretativo, se utilizó la comprensión de la alimentación como un acto biosociocultural, de la SAN y de la Educación Alimentaria y Nutricional (EAN). Las acciones educativas fueron realizadas en el municipio de Acrelandia, Acre, con cuarenta y cinco ACS y dos nutricionistas, y desarrolladas en cuatro etapas: diagnóstico, planeación, ejecución y evaluación. En esta experiencia de investigación se produjeron cuatro acciones por medio de talleres educativos y talleres culinarios. Por lo identificado, parece fundamental reflexionar sobre las prácticas de la EAN, teniendo en cuenta al equipo multidisciplinario, así como también al usuario y al territorio, esto como una propuesta para realizar el cuidado nutricional desde la perspectiva de la Atención Primaria de Salud.

Palabras-claves: Atención primaria de salud. Educación alimentaria y nutricional. Hábitos alimentarios. Promoción de la salud. Ecosistema amazónico.

\section{Introdução}

A Organização das Nações Unidas para a Alimentação e Agricultura (FAO) traz as ações de educação alimentar e nutricional (EAN) como estratégias para sensibilização, atitudes e habilidades em torno de uma alimentação saudável. Além de encorajar organizações internacionais, governos, o setor privado e sociedade civil (organizações não governamentais, indivíduos e comunidades) a se engajarem na promoção e realização dessas ações ${ }^{1,2}$.

A EAN, em seu sentido mais amplo apresenta três componentes importantes a serem considerados em ações educativas: a prestação de informações por meio de estratégias de comunicação (campanhas de informação, aconselhamento dietético em ambientes de serviços de saúde), o fornecimento de habilidades que permitem os consumidores a agirem sobre a informação fornecida (culinária, compreensão de rótulos de alimentos) e um ambiente alimentar que proporcione escolhas saudáveis, com disponibilidade diversa de alimentos e marketing de alimentos regulado ${ }^{1,2}$. 
ISSN 2179-6750

As Doenças Crônicas Não Transmissíveis (DCNTs) como obesidade, hipertensão, diabetes são atualmente um sério problema de saúde pública global, as quais tem sido responsáveis por $80 \%$ das mortes que ocorrem nos países em desenvolvimento ${ }^{1,3}$ e um dos principais fatores possíveis para intervenções de promoção, prevenção e tratamento dessas doenças é a alimentação ${ }^{4,5}$.

No Brasil, a crescente prevalência de sobrepeso e obesidade constitui um dado alarmante dentro do perfil epidemiológico da população brasileira, 54\% da população maior de 18 anos vivenciam essa situação ${ }^{4}$. Em 2013, de acordo com pesquisa de Vigilância de Fatores de Risco e Proteção para Doenças Crônicas por Inquérito Telefônico (VIGITEL), cerca de 50\% dos brasileiros maiores de 18 anos apresentaram excesso de peso e $17,5 \%$ de obesidade ${ }^{6}$.

No cenário das políticas públicas brasileiras, existem documentos importantes que visam orientar as ações de saúde relacionadas à alimentação para o enfrentamento do sobrepeso e da obesidade. Dentre eles encontra-se o Plano Nacional de Segurança Alimentar e Nutricional, que menciona os agravos e as doenças consequentes da insegurança alimentar e nutricional (IAN), e direciona para a criação de um plano voltado para o controle e a prevenção dessas morbidades ${ }^{7,8}$. No mesmo direcionamento, o Brasil apresenta a Política Nacional de Alimentação e Nutrição (PNAN) e o Plano de Ações Estratégicas para o Enfrentamento das Doenças Crônicas não Transmissíveis, os quais explicitam a importância da alimentação para o controle do sobrepeso e da obesidade por se constituírem fatores de risco para outras doenças que compõem esse conjunto, como diabetes e hipertensão ${ }^{9,10}$.

Em 2014, o Ministério da Saúde divulgou os Cadernos de Atenção Básica n. 12, um documento referente às estratégias de cuidado para indivíduos com obesidade, a fim de subsidiar os profissionais da saúde em relação à promoção do cuidado integral, com foco no manejo alimentar e nutricional $^{11}$.

Além disso, publicou a portaria $n^{\circ} 424$ de 19 de março de 2013, que redefine as diretrizes para a organização da prevenção e do tratamento do sobrepeso e da obesidade como linha de cuidado prioritária da Rede de Atenção à Saúde das Pessoas com Doenças Crônicas. Nesta normativa encontram-se informações sobre o fortalecimento e qualificação para a atenção nutricional mediante o estabelecimento de fluxos de referência e contrarreferência dentro dos diferentes pontos de atenção da rede. Define também, a organização de sistemas de apoio e dos processos de trabalho para que, propiciando o funcionamento harmônico e integral dos serviços de saúde, o acesso do usuário a esses espaços seja facilitado ${ }^{12}$.

De acordo com a Política Nacional de Atenção Básica e a PNAN, esses serviços e ações de cuidado à saúde (como a atenção nutricional, neste exemplo) devem acontecer de forma 248 
ISSN 2179-6750

intersetorial, em rede, com trabalho em equipe, cabendo aos seus membros identificar parceiros e recursos na comunidade. Essas características visam potencializar a atuação entre os diferentes níveis de atenção que compõe o Sistema Único de Saúde (SUS), como por exemplo, os ambulatórios especializados, a rede de urgência e emergência, centros de atenção psicossociais, os serviços e instituições componentes do Sistema Único de Assistência Social (SUAS) ${ }^{9}$.

No que se refere aos processos de trabalho acerca da alimentação e da nutrição, a atuação do nutricionista configura-se como um eixo central das ações de promoção da saúde produzidas com EAN, que a partir do aprimoramento da sua atuação profissional faz-se possível uma melhor articulação intra e intersetorial, de trabalho, de matriciamento e de trabalho compartilhado com os demais profissionais da equipe que compõem a Estratégia Saúde da Família, como o agente comunitário de saúde (ACS) e a própria comunidade ${ }^{2,13}$.

Diante disso, desenvolver mecanismos permanentes de qualificação técnica utilizando diversas estratégias educativas (caminhos metodológicos) para o aperfeiçoamento das práticas profissionais na Atenção Primária à Saúde (APS), torna-se fundamental para a atuação, gestão e atenção do cuidado em saúde. Visto que, as práticas educativas em alimentação realizadas neste espaço são pouco exploradas na perspectiva dos profissionais em saúde, principalmente numa abordagem de EAN para a realização da Segurança Alimentar e Nutricional (SAN) e do Direito Humano à Alimentação Adequada (DHAA) ${ }^{14,15}$. Assim, este relato de experiência tem o objetivo de apresentar as ações educativas realizadas com agentes comunitários de saúde e nutricionistas da equipe Estratégia Saúde da Família da APS, na região da Amazônia Ocidental Brasileira.

\section{Métodos}

Este relato de experiência é uma síntese sistematizada da vivência produzida no projeto “Ações educativas para promoção da alimentação saudável em comunidades de baixa renda: estudo piloto em Acrelândia, Acre" realizado em julho de 2011. O projeto foi aprovado pelo Comitê de Ética em Pesquisa da Faculdade de Saúde Pública (OF.COEP/266/11-protocolo nº. 2272) e contou com financiamento do Conselho Nacional de Desenvolvimento Científico e Tecnológico- CNPq (Proc 559461/2010-0).

O local da vivência foi o município de Acrelândia situado no noroeste do estado do Acre, a $112 \mathrm{~km}$ a leste da capital Rio Branco (Figura 1). No período da pesquisa, o município tinha uma população de 12.538 habitantes, dos quais 5.916 (47,2\%) residiam na área urbana e 6.622 na área rural $(52,8 \%)^{16}$.

Acrelândia é uma área típica de fronteira agrícola, destacando-se na extração da madeira, no 
ISSN 2179-6750

cultivo de café, banana, algodão, extração de castanha do Brasil e açaí. Além da produção permanente de coco-da-baía, laranja, limão, mamão, manga, maracujá, palmito, tangerina, urucum, pimenta-do-reino e a produção temporária de abacaxi, arroz, cana de açúcar, feijão, mandioca, melancia e milho ${ }^{17}$.

Figura 1. Localização geográfica do município de Acrelândia - Acre, Brasil. Região da Amazônia Ocidental Brasileira.

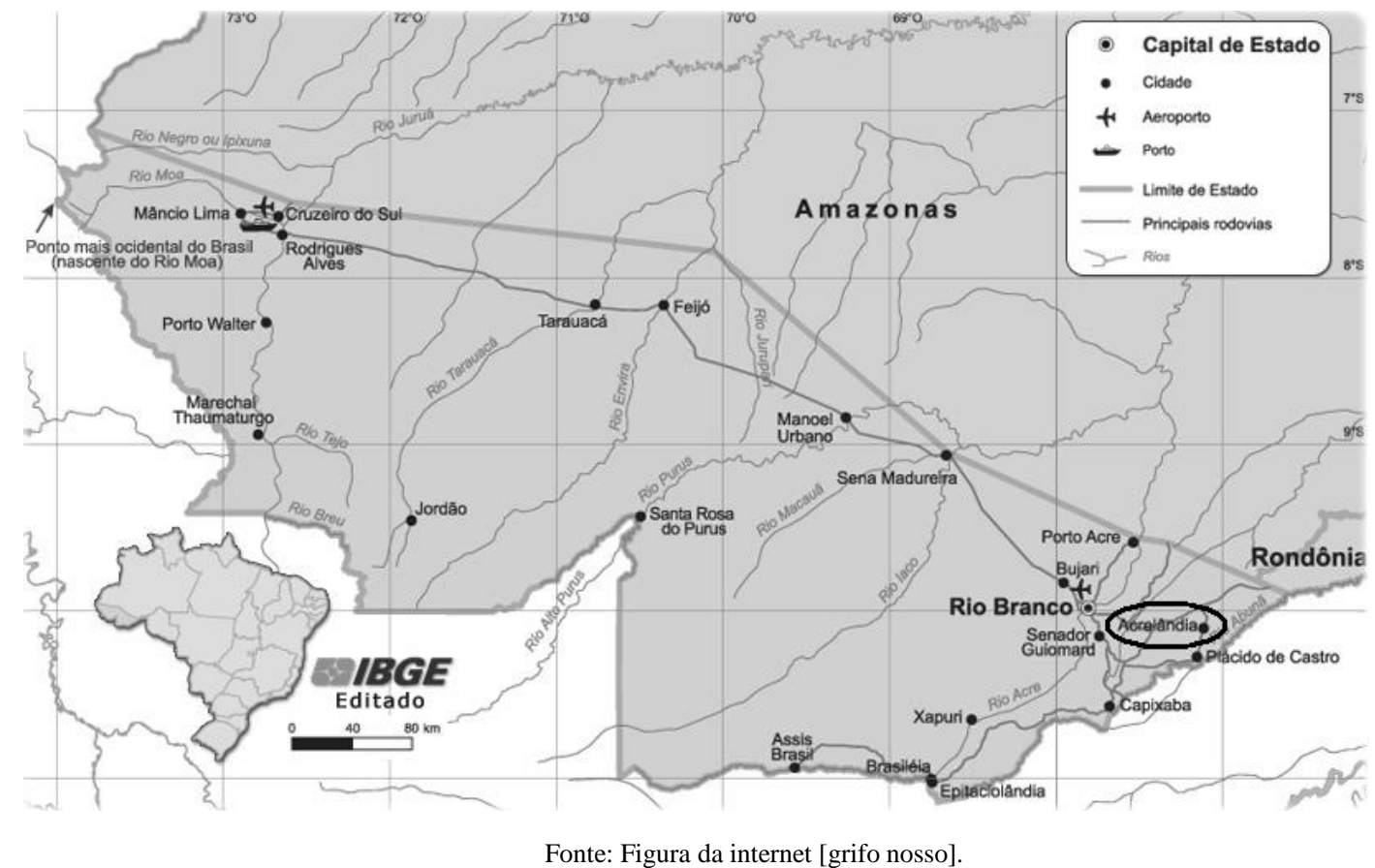

A APS do município, no período da pesquisa, contava com $100 \%$ de cobertura do Programa Saúde da Família, com cinco unidades de Estratégia Saúde da Família (ESF) e um Núcleo de Apoio à Saúde da Família (NASF). Dentre as estruturas de assistência social havia um Centro de Referência Social (CRAS) que atendia 915 crianças, idosos e suas famílias, e uma unidade do Programa de Erradicação do Trabalho Infantil - PETI que atendia 240 crianças e adolescentes.

$\mathrm{Na}$ realização da pesquisa de campo havia quatro pesquisadores: três nutricionistas e um biomédico. Inicialmente houve reuniões com o secretário municipal de saúde e a equipe de ESF para apresentação e discussão das ações educativas a serem desenvolvidas. Na reunião com a equipe de ESF estavam presentes três profissionais, a coordenadora da atenção básica do município, uma nutricionista e um educador físico, além da nutricionista da educação, responsável pela alimentação escolar.

Nesta reunião foi possível identificar alguns grupos que já eram produzidos regularmente e 
ISSN 2179-6750

apresentavam características importantes para a realização das ações educativas. Após a reunião, decidimos trabalhar com o grupo de ACS. O motivo da escolha foi a demanda apresentada pela nutricionista do NASF, quanto a necessidade de capacitação sobre o tema alimentação e nutrição, principalmente na abordagem de questões relacionadas à hipertensão e diabetes junto à população.

Quadro 1. Etapas de construção das ações educativas realizada com ACS e nutricionistas da Atenção Primária à Saúde, na Região da Amazônia Ocidental Brasileira. Acrelândia, Acre, Brasil. 2011.

\begin{tabular}{|c|c|c|c|c|}
\hline Etapa 1 & Necessidade local & Público alvo & $\begin{array}{l}\text { Agentes chave para } \\
\text { execução da ação }\end{array}$ & $\begin{array}{l}\text { Local de realização } \\
\text { das ações }\end{array}$ \\
\hline & $\begin{array}{l}\text { Dificuldade dos ACS } \\
\text { em abordarem } \\
\text { questões relacionadas } \\
\text { à hipertensão e } \\
\text { diabetes junto à } \\
\text { população }\end{array}$ & $\begin{array}{c}\mathrm{ACS} \text { e } \\
\text { nutricionistas }\end{array}$ & $\begin{array}{c}\text { Coordenadora da } \\
\text { Atenção Básica, } \\
\text { nutricionistas, secretário } \\
\text { de saúde }\end{array}$ & $\begin{array}{l}\text { Espaço do Centro de } \\
\text { Cultura e Florestania }\end{array}$ \\
\hline \multirow[t]{2}{*}{$\begin{array}{c}\text { Etapa 2 } \\
\text { Planejamento } \\
\end{array}$} & $\begin{array}{c}\text { Convite aos } \\
\text { participantes }\end{array}$ & Objetivos & Metodologia & Materiais e recursos \\
\hline & $\begin{array}{l}\text { Via coordenadoria da } \\
\text { Atenção Básica e } \\
\text { nutricionista }\end{array}$ & $\begin{array}{l}\text { Sensibilizar; } \\
\text { Reconhecer } \\
\text { alimentos locais e } \\
\text { resgatar a memória } \\
\text { dos participantes } \\
\text { sobre alguns } \\
\text { alimentos; } \\
\text { Promover interação } \\
\text { e prática; Construir } \\
\text { conhecimento a } \\
\text { respeito das } \\
\text { doenças crônicas } \\
\text { não transmissíveis }\end{array}$ & $\begin{array}{l}\text { Estratégias pedagógicas: } \\
\text { roda de conversa, } \\
\text { palestra, oficina } \\
\text { culinária. } \\
\text { Temas: alimentação } \\
\text { saudável, alimentos } \\
\text { locais, doenças crônicas } \\
\text { não transmissíveis. } \\
\text { Tempo de duração: oito } \\
\text { horas }\end{array}$ & $\begin{array}{c}\text { Apoio financeiro da } \\
\text { coordenação da } \\
\text { Atenção Básica, } \\
\text { do projeto de pesquisa, } \\
\text { doação comunitária de } \\
\text { alimentos locais } \\
\text { produzidos nos quintais } \\
\text { da população; } \\
\text { Recursos humanos para } \\
\text { preparação: equipe de } \\
\text { pesquisadores }\end{array}$ \\
\hline \multirow[t]{2}{*}{$\begin{array}{c}\text { Etapa 3 } \\
\text { Execuçãao } \\
\end{array}$} & $\begin{array}{c}\text { Momento de } \\
\text { sensibilização }\end{array}$ & $\begin{array}{c}\text { Momento de } \\
\text { reconhecimento }\end{array}$ & Momento prática & $\begin{array}{l}\text { Momento de } \\
\text { Construção de } \\
\text { Conhecimento }\end{array}$ \\
\hline & $\begin{array}{l}\text { Café da manhã com } \\
\text { preparo de alimentos } \\
\text { locais }\end{array}$ & $\begin{array}{l}\text { Reconhecimento de } \\
\text { alimentos locais e } \\
\text { resgate da memória } \\
\text { alimentar }\end{array}$ & $\begin{array}{l}\text { Preparo culinário de um } \\
\text { almoço coletivo pelos } \\
\text { próprios participantes }\end{array}$ & $\begin{array}{l}\text { Orientações por meio } \\
\text { de palestra sobre } \\
\text { diabetes, hipertensão, e } \\
\text { rotulagem de alimentos, } \\
\text { com apresentação e } \\
\text { manuseio de temperos } \\
\text { prontos industrializados }\end{array}$ \\
\hline $\begin{array}{c}\text { Etapa } 4 \\
\text { Avaliação } \\
\text { Informal }\end{array}$ & \multicolumn{4}{|c|}{ momento de expressão do olhar avaliativo dos participantes } \\
\hline
\end{tabular}

Após esse momento exploratório, realizamos a "Oficina educativa e culinária para alimentação saudável” com 45 ACS e duas nutricionistas (NASF e alimentação escolar). A oficina 
ISSN 2179-6750

foi desenvolvida em quatro etapas: diagnóstico, planejamento, execução e avaliação.

Na etapa 1 - Diagnóstico: identificamos a necessidade local, o público alvo, os agentes chave e o local para execução da ação. Na etapa 2 - Planejamento: elaboramos o convite dos participantes, os objetivos da ação, a metodologia a ser desenvolvida, os materiais e recursos necessários. Na etapa 3 - Execução: as ações foram conduzidas em quatro momentos - primeiro um café da manhã preparado com alimentos locais por uma das pesquisadoras da equipe de campo, seguida de uma roda de conversa com o tema "Hortas Esquecidas", uma oficina culinária "Almoço Coletivo" preparado pelos próprios participantes e uma palestra com o tema "Doenças Crônicas Não Transmissíveis: Hipertensão e Diabetes”. Na etapa 4 - Avaliação Informal: foi criado um momento para a expressão do olhar avaliativo dos participantes (Quadro 1).

A fundamentação metodológica para a construção das ações educativas teve como base a investigação pedagógica, de abordagem qualitativa ${ }^{18}$. Neste tipo de investigação o pesquisador se posiciona como um praticante que pretende utilizar uma abordagem interventiva (quanti ou qualitativa) para otimizar aquilo que é objeto de sua prática e assim auxiliando os participantes da ação a explorarem a sua própria comunidade ${ }^{18}$. Para esta intervenção tomou-se como conceito de alimentação, aquele apresentado por Canesqui ${ }^{19}$ que considera a alimentação como um ato social, cultural e biológico, um conjunto de hábitos e substâncias que o homem usa, não só em relação às suas funções vitais, mas também como um elemento da sua cultura para manter ou melhorar a sua saúde.

Em complemento ao conceito acima descrito, a compreensão do conceito de $\mathrm{SAN}^{20}$ e de EAN $^{2}$ foram fundamentais para nortear as ações, eles compuserem esse processo de aprendizagem constante e dinâmico que envolve questões complexas como cultura, economia, relações, território, crenças, que visa o desenvolvimento da capacidade da população em utilizar os recursos disponíveis e reconhecer caminhos par exercer práticas alimentares saudáveis ${ }^{15}$.

Visando a disseminação das ações educativas produzidas foi criado o blog "Frutos do Norte: alimentação e nutrição em saúde", com o objetivo de utilizá-lo como tecnologia social, fruto dessa intervenção ${ }^{21,22}$. No blog estão disponíveis fotos, vídeos produzidos por um jornalista local e diversas informações sobre o projeto de pesquisa e a equipe de pesquisadores.

\section{Resultados e Discussão}

Nesta experiência de pesquisa foram produzidas quatro ações educativas com 45 ACS e duas nutricionistas que compõem a equipe de ESF da APS do município de Acrelândia, Acre. O local de execução das ações foi o Centro de Cultura e Florestania, espaço utilizado para a realização de 
ISSN 2179-6750

atividades coletivas no município. As ações foram desenvolvidas por meio de oficinas educativas e oficinas culinárias realizadas em um mesmo dia, em cinco momentos, com duração total de oito horas (Quadro 2).

Quadro 2. Ações educativas realizadas com ACS e nutricionistas da Atenção Primária à Saúde, na Região da Amazônia Ocidental Brasileira. Acrelândia, Acre, Brasil. 2011.

\begin{tabular}{|l|c|c|c|}
\hline \multicolumn{1}{|c|}{ Ações educativas } & Objetivo & Estratégia pedagógica & Recurso \\
\hline $\begin{array}{l}\text { 1) Café da manhã com } \\
\text { alimentos locais }\end{array}$ & Sensibilizar & Degustação & $\begin{array}{l}\text { Preparações culinárias } \\
\text { com alimentos locais }\end{array}$ \\
\hline 2) Hortas Esquecidas & $\begin{array}{c}\text { Sensibilizar, } \\
\text { reconhecer, resgatar } \\
\text { hábitos e práticas } \\
\text { alimentares esquecidas }\end{array}$ & Roda de conversa & $\begin{array}{c}\text { Audiovisual: } \\
\text { computador, tela de } \\
\text { projeção e datashow }\end{array}$ \\
\hline $\begin{array}{l}\text { 3) Almoço Coletivo } \\
\text { 4) Orientações sobre } \\
\text { doenças crônicas não } \\
\text { transmissíveis }\end{array}$ & $\begin{array}{c}\text { Interação e prática } \\
\text { conhecimento }\end{array}$ & Oficina culinária & Preparo de alimentos \\
\hline $\begin{array}{l}\text { 5) Espaço de avaliação } \\
\text { informal }\end{array}$ & momento produzido para a expressão do olhar avaliativo dos participantes \\
\hline
\end{tabular}

Esta experiência possibilitou compreender na prática a atuação em nutrição, na perspectiva interdisciplinar da saúde, que esta ancorada na capacidade de integrar abordagens biológicas, sociais, políticas e ambientais que contribuam para a construção de respostas que favoreçam a melhoria das condições de vida das populações, na interface alimento-sujeito-sociedade ${ }^{23}$. Nesse sentido, a EAN constituiu um suporte essencial para integrar diferentes estratégias pedagógicas com o objetivo de promoção da saúde, especialmente para ações de promoção da SAN que buscam a garantia do DHAA $^{2,15}$.

No contexto da APS é estimulada a realização de práticas educativas tendo em vista à educação em saúde enquanto prática social de (re)conhecimento de sujeitos e construção de novos saberes ${ }^{24,25}$. Para isso, o uso de estratégias educativas que valorizam a participação coletiva, o perfil da população, a disponibilidade de recursos humanos e materiais, além das crenças e conhecimentos dos sujeitos envolvidos na ação parecem contribuir para a produção de espaços promotores de saúde ${ }^{26}$ de uma forma mais facilitada.

No passo-a-passo da ação, construiu-se o primeiro momento das ações educativas através de uma vivência sensorial com um café da manhã preparado por uma das pesquisadoras da equipe, com alimentos regionais produzidos localmente. Essa ação buscou sensibilizar os participantes quanto ao preparo, reconhecimento e degustação dos alimentos locais. Os alimentos preparados para essa intervenção foram pão caseiro de banana da terra, suco de carambola, cuscuz de milho e 
ISSN 2179-6750

sushi de tapioca (tapioca molhada no leite de coco e recheada com banana da terra).

As discussões no café da manhã geraram alguns comentários. Foi uma satisfação e ao mesmo tempo, um despertar de convívio, expresso de maneira sutil nas falas dos participantes:

[...] “mas o que é isso, suco de carambola?” [...]

[...] "nossa, tapioca com banana? hum que delícia! O que mais que tem? Erva doce? e esse gostinho de coco?" [...]

"E esse pão? que legal, na folha de bananeira...ficou bonito!"

[...] “pão com manteiga? e café com leite!” [...]

[...] "e isso...é cuscuz? foi você quem fez? você quem preparou tudo isso? Ficou muito gostoso! Pode comer mais?...” [...]

"Que tanto de açúcar coloca no café?"

"Achei o suco meio sem doce! Pode colocar mais açúcar?" "Melhor não, né, eles já fizeram com a quantidade necessária!’

Nessa vivência sensorial pôde-se observar a sensibilização dos participantes durante o ato de comer assim como o reconhecimento dos alimentos. Desse modo, utilizar o café da manhã preparado com alimentos locais, como estratégia pedagógica de sensibilização, despertou curiosidade em saber como eles foram preparados, qual foi o local de aquisição desses alimentos: os quintais de Acrelândia.

O conceito de alimentação adequada é permeado pelos princípios aplicados à nutrição: variedade, equilíbrio, moderação, prazer (sabor), envolvendo a dimensão sensorial para a realização do que chamamos de alimentação saudável. De acordo com a III Conferência Nacional de SAN realizada em 2007, uma alimentação adequada é:

\footnotetext{
"a realização de um direito básico, com garantia ao acesso permanente e regular, socialmente justa, a uma prática alimentar adequada aos aspectos biológicos e sociais dos indivíduos, de acordo com o ciclo de vida e as necessidades alimentares especiais, pautada no referencial tradicional local. Deve atender aos princípios da variedade, equilíbrio, moderação, prazer (sabor), dimensões de gênero e etnia, e formas de produção ambientalmente sustentáveis, livre de contaminantes físicos, químicos e biológicos e organismos geneticamente modificados" ${ }^{27}$.
}

Essa construção do que vem a ser "comida de verdade" foi tema central de debate da V Conferência Nacional de Segurança Alimentar e Nutricional realizada em 2015, espaço que busca ampliar o diálogo entre os diferentes atores sociais como profissionais de saúde e visa fortalecer 
ISSN 2179-6750

ações e políticas públicas de garantia do DHAA. Apesar de haver esse espaço para socialização de saberes sobre alimentação, SAN e políticas públicas, os ACS e demais profissionais que compõem a ESF, considerados elementos fundamentais para a promoção em saúde, parecem necessitar de educação permanente voltada para qualificação política e científica das dimensões da SAN ${ }^{14,28,29}$.

A realização de ações educativas em alimentação e nutrição exigem um diagnóstico e um planejamento conectados com a realidade local ${ }^{15,30}$. No entanto, é necessário estar preparado para a elaboração e reorganização da ação planejada mediante a dinâmica muitas vezes apresentada pela realidade. Isso foi vivenciado na presente experiência. Um possível caminho utilizado foi o compartilhamento das situações imprevistas e a construção participativa como proposta, como é relatado no trecho a seguir.

Logo após o café da manhã, surgiu a seguinte notícia:

"Teremos que terminar a oficina neste período da manhã, pois a pessoa que vinha ajudar no preparo do almoço dos agentes não pode vir!"

Pensamos imediatamente: E agora? A festa acabou... E rapidamente nos surgiu a ideia: vamos todos juntos preparar o almoço, somente os ACS e nutricionistas, sem ajudante! Manteremos a programação das ações. Temos todos os ingredientes? O que falta? O cardápio está feito. Desse modo, enquanto pesquisadora e nutricionista, coloquei-me em ação. No entanto, um participante fez o seguinte comentário:

"Bom, agora você conversa com os agentes, acho que eles não vão querer, será? Cozinhar? Ah! eles preferem ir embora!"

Diante deste cenário, foi conversado com todos os participantes e explicado a situação de não contarmos mais, com um ajudante para preparar o almoço e assim, lançamos a questão: vocês aceitam produzir um almoço coletivo, só nós? Para nossa surpresa, todos os ACS levantaram os braços em sinal de afirmação e se dispuseram a preparar o nosso almoço. Foi uma belíssima experiência!

Então, retomamos e demos início à segunda ação educativa, a roda de conversa: "Hortas esquecidas". Neste momento, nossas experiências pessoais reproduziam-se em mim como em um filme, elas passavam uma a uma em minha mente.

No início da roda de conversa foi possível construir um momento no qual, cada agente pudesse expressar através da fala, o seu nome, a região de atuação na ESF e a sua percepção a 255 
ISSN 2179-6750

respeito do papel de um ACS junto à comunidade. Deste momento, destacamos as seguintes falas:

[...] "somos um elo, uma ponte entre o conhecimento sobre saúde e a população"[...]

[...] "é ser alguém em quem as pessoas confiam, pois entramos nas casas das pessoas para dizer algo importante" [...]

[...] "ter a responsabilidade com a função que nos foi confiada" [...]

Foi possível observar a importância da criação de momentos como este, nos quais se proporciona o espaço para os ACS localizarem-se como sujeito em ação ou participante central do processo. Usar de abordagens e recursos educacionais problematizadores que favoreçam a construção do saber alimentar pelo sujeito é um dos marcos que orientam como devem ser realizadas as ações educativas segundo a EAN. É pertinente lembrar que, no contexto da realização do DHAA, a prática permanente, transdisciplinar e multiprofissional, são fundamentais para favorecer o diálogo, interações e os significados que compõem o comportamento alimentar ${ }^{2}$.

Para a roda de conversa utilizou-se recursos áudio visuais (tela de projeção e datashow) para a projeção das imagens de alguns alimentos, com o propósito de sensibilizar e resgatar hábitos e práticas alimentares esquecidos.

A roda de conversa "Hortas Esquecidas" possibilitou o resgate da lembrança de alimentos e momentos vivenciados durante a infância e a adolescência. Além disso, foi possível observar a troca de experiências entre os ACS sobre tipos de frutas, legumes e verduras pouco utilizados na culinária local, no entanto, presentes e produzidos nas hortas domésticas, hortas comunitárias e hortas comerciais da área urbana do município. Isso sinaliza a legitimidade dos diferentes saberes presentes na alimentação brasileira, com suas particularidades regionais. Uma expressão oriunda da cultura alimentar e do intercâmbio entre os diversos povos que formaram nossa nação ${ }^{9}$.

Nessa ação também se observou a identificação e o reconhecimento dos ACS a cerca das espécies comestíveis ilustradas e apresentadas, as Plantas Comestíveis Não Convencionais (PANCs): beldroega, bertalha, caruru, taioba, serralha, ora-pro-nobis, jacatupé, orelha de padre, cará-moela entre outros ${ }^{31,32}$. Essas plantas estão presentes nos saberes populares das diversas regiões brasileiras, além de fazerem parte de preparações culinárias.

Após a roda de conversa, todos os participantes foram para a cozinha do Centro de Cultura e Florestania realizarem a oficina culinária. O ambiente se configurou em uma grande cozinha comunitária onde alguns se dedicavam a picar carne, outros a lavar as hortaliças e dentre tantos compartilhamento de tarefas o almoço coletivo foi produzido. O cardápio foi arroz, estrogonofe de carne, batata palha, salada de alface, tomate e rúcula. Durante esse momento, apareceram algumas 
ISSN 2179-6750

crianças que comeram as carambolas que sobraram nas cestas do café da manhã. Com o almoço pronto e a mesa preparada pelos participantes do sexo masculino, todos saborearam a delícia da atitude, da partilha, da coragem, da iniciativa e da união. Tudo produto de uma construção coletiva.

$\mathrm{Na}$ oficina culinária, durante o preparo do almoço predominou a interação e o compartilhamento de saberes, do mesmo modo, foi possível observar esses elementos durante o momento da refeição, sinalizando que a alimentação é sim um espaço e um ato social, conforme afirma Canesqui ${ }^{19}$. A preparação coletiva do almoço promoveu a socialização do conhecimento e gerou grupalidade.

A partir dessa experiência, é possível discutir a culinária como ferramenta que potencializa a alimentação enquanto espaço social alimentar proporcionando um ambiente de liberdade no qual a relação entre o biológico e o cultural ganha concretude ${ }^{33}$.

Após o almoço, foi realizada a palestra "Doenças Crônicas não Transmissíveis: diabetes e hipertensão" com orientações e distribuição de material educativo (folheto) sobre o assunto. Esta ação educativa teve o intuito de explorar os conhecimentos prévios dos participantes e de sensibilizá-los quanto à importância de ter uma alimentação saudável, utilizando temperos e ervas naturais ao invés de temperos prontos.

A palestra iniciou com uma salva de palmas para todos que preparam o almoço coletivo e em seguida começaram as orientações e as exposições dialogadas. Para exemplificar a quantidade elevada de sódio presente nos temperos prontos industrializados utilizamos embalagens e também trabalhamos a leitura e a compreensão dos rótulos.

Nessa última ação observamos o pouco conhecimento dos participantes sobre a quantidade recomendada de ingestão de sódio e a quantidade elevada presente nos temperos prontos industrializados. Isso destaca a necessidade local apontada pela nutricionista do NASF, na etapa diagnóstica das ações educativas.

A prevalência de hipertensão decorrente do consumo excessivo de sódio, assim como a diabetes e o excesso de peso tem aumentado entre a população brasileira. Estas têm sido um conjunto de doenças que se tornaram uma das principais prioridades da saúde no Brasil, por ser fonte de diversas doenças e causadoras de mobirmortalidade entre grande parte da população mais pobre $^{5,10}$.

Diante disso, essa experiência reitera a responsabilização que o setor saúde deve comprometer-se para construção de novas estratégias de enfrentamento dessa situação. Articular as ações já desenvolvidas para a organização do cuidado em alimentação e nutrição na APS (vigilância alimentar e nutricional, promoção de práticas alimentares saudáveis, cuidado integral dos agravos 
ISSN 2179-6750

nutricionais) ${ }^{34,35}$ com experiências exitosas como esta que foi relatada parece ser uma contribuição bem vinda.

Por fim, produziu-se um momento de avaliação informal das ações educativas realizadas. No olhar avaliativo dos coordenadores, o grupo conseguiu relacionar as ações desenvolvidas, além de perceberem a importância do reconhecimento e do consumo dos alimentos regionais produzidos localmente para uma alimentação saudável. Nas falas dos participantes foi reconhecido o seu papel de multiplicador do conhecimento produzido:

"Então, a gente vai estar mais capacitado para entender as nossas famílias, nas nossas áreas”.

"É muito importante a gente ter essa informação para tá passando para população, para comunidade"

"Nós vamos servir de elo, nós vamos ser canal, uma ponte entre nós e o que vamos aprender aqui para transmitir na sociedade”

Considerar a experiência do outro em práticas educativas nos serviços de saúde permite uma melhor apropriação social do cuidado, pois a memória afetiva dos envolvidos é acessada e o produto do aprendizado é conduzido às expectativas e aos projetos de vida dos partícipes da ocasião (usuários e também dos profissionais) ${ }^{36}$. Tradicionalmente se observa que, no cenário da APS, predomina uma postura do profissional de saúde que tende a ser legitimada por uma fala socialmente autorizada, o que torna as relações com os usuários assimétricas e prejudica o (di)á $\operatorname{logo}(\mathrm{di}=$ dois; $\operatorname{logo}=$ conhecimento $)$. Mesmo com tentativas mais dialógicas, as discussões tendem a ser guiadas através da lógica de produção de sentidos, atribuída unicamente pelo profissional, o que produz uma compreensão do fenômeno distanciada da perspectiva dos usuários 37.

Nesse sentido, este relato de experiência teve como finalidade refletir sobre a necessidade de aprofundar o debate sobre as práticas atuais de EAN realizadas na APS e produzir a divulgação de uma experiência bem sucedida, tendo como perspectiva que essa é uma das ações que podem proporcionar sujeitos sociais ativos e autônomos em saúde, conforme preconiza o princípio da competência cultural tão necessária à qualidade da prestação dos serviços de atenção primária no Brasil $^{2}$.

Pelo identificado na experiência descrita parece fundamental repensar as práticas de EAN considerando a equipe multiprofissional, bem como o usuário e o território. Considerar estes três 
ISSN 2179-6750

elementos foi essencial para o sucesso da intervenção proposta, o que poderia ser utilizado como uma proposta para se realizar o cuidado nutricional em saúde na perspectiva da APS.

\section{Considerações finais}

Promover educação alimentar e nutricional através de oficinas educativas e culinárias para capacitação permanente de profissionais de saúde em alimentação e nutrição possibilitou explorar questões importantes para a APS, como o vínculo, a interação, a prática, a aproximação com o cotidiano, considerando a valorização da cultura local, a identidade e o ambiente alimentar da região.

As ações educativas visaram contribuir para a formação e fortalecimento do papel dos profissionais de saúde em sua atuação cotidiana no âmbito da alimentação e nutrição, especialmente nutricionistas e ACS, explorando a diversidade de alimentos regionais. Tentou-se resgatar elementos cognitivos, práticos e de construção de uma educação mais solidária e colaborativa que se aproxima do cotidiano de trabalho dos profissionais de saúde.

Diante disso, pistas ou caminhos puderam se construir para a realização de ações educativas que utilizam a EAN para a promoção do DHAA, como, por exemplo, a necessidade de se realizar bem a fase de diagnóstico e planejamento para a execução da ação. Outro elemento que parece fundamental é reconhecer que a aplicação ou reprodução de um conhecimento técnico é diferente da construção de estratégia, na qual se busca conhecer o ambiente, o espaço, a necessidade do outro e só então, em conjunto produzir uma ação.

$\mathrm{Na}$ questão da alimentação saudável, outro caminho ficou evidente, o desafio de produzir ações educativas em meio a um ambiente alimentar avassalador e repleto de alimentos ultraprocessados, cujo marketing estimula o consumo e (re)produz práticas alimentares pouco saudáveis. Diante disso, urge retomar práticas de consumo de alimentos tradicionais, esquecidos, produzidos cotidianamente nas hortas domésticas. Retornar ao passado e relembrar as práticas vivenciadas na infância, na adolescência mostra que hábitos alimentares mais saudáveis já foram vivenciados e é preciso resgatá-los.

Dentre os desafios apresentados neste relato de experiência está o planejamento das ações a partir da realidade apresentada e dos alimentos disponíveis no local. Além do uso da culinária como ferramenta para a apresentação dos alimentos regionais, a fim de produzir sensibilização, atitudes e habilidades entre os participantes com o objetivo de promover a alimentação saudável.

Tratou-se de uma atividade instigante e desafiadora para todos os profissionais de saúde, com destaque para os nutricionistas quando se deparam com o descompasso entre o cenário 
ISSN 2179-6750

disposto (de abundância de recursos alimentícios locais) e no tempo histórico vigente (de alimentações rápidas, hipercalóricas e ultraprocessadas). Para experiências futuras que venham a desenvolver ações educativas sugere-se que seja elaborada uma avaliação processual formativa, na qual haja a manifestação de todos os participantes: profissionais e coordenadores da ação.

\section{Agradecimentos}

À Coordenação de Aperfeiçoamento de Pessoal de Nível Superior - Capes, pela bolsa de doutorado concedida à autora do manuscrito. À Secretaria Municipal de Acrelândia, aos profissionais de saúde e a população local. Ao Conselho Nacional de Desenvolvimento Científico e Tecnológico- CNPq (Proc 559461/2010-0) pelo financiamento do projeto de pesquisa, no qual se produziu a vivência.

\section{Referências}

1. Food and Agriculture Organization of the United Nations - FAO [homepage na internet]. Promoting healthy diets through nutrition education and changes in the food environment: an international review of actions and their effectiveness 2013. Rome; 2013. [atualizado em 28 jul. 2016; acesso em 14 abr 2015]. Disponível em: www.fao.org/publications

2. Brasil. Ministério do Desenvolvimento Social e Combate à Fome. Secretaria Nacional de Segurança Alimentar e Nutricional. Marco de referência de Educação Alimentar e Nutricional para as Políticas Públicas. Brasília: MDS; 2012.

3. Popkin BM. The nutrition transition and its health implications in lower-income countries. Public Health Nutr. 1998; 1:5-21.

4. World Health Organization - WHO. Global Status Report on noncommunicable diseases 2014: "Attaining the nine global noncommunicable diseases targets; a shared responsibility". Geneva; 2014. [atualizado em 28 jul. 2016; acesso em 28 jul 2016]. Disponível em: http://apps.who.int/iris/bitstream/10665/148114/1/9789241564854_eng.pdf

5. Schmidt MI, Duncan BB, Azevedo e Silva G, Menezes AM, Monteiro CA, Barreto SM, Chor D, Menezes PR. Doenças crônicas não transmissíveis no Brasil: carga e desafios atuais. Lancet. 2011; 377(9781):1949-1961.

6. Brasil. Ministério da Saúde. Secretaria de Vigilância em Saúde. Departamento de Vigilância de Doenças e Agravos não Transmissíveis e Promoção da Saúde. Vigitel Brasil 2013. Brasília: MS; 2014.

7. Brasil. Ministério do Desenvolvimento Social e Combate à Fome. Câmara Interministerial de Segurança Alimentar e Nutricional. Plano Nacional de Segurança Alimentar e Nutricional: 2012/2015. Brasília: MDS; 2011. 
ISSN 2179-6750

8. Brasil. Ministério do Desenvolvimento Social e Combate à Fome. Câmara Interministerial de Segurança Alimentar e Nutricional. Plano Nacional de Segurança Alimentar e Nutricional: 2016/2019. Brasília: MDS; 2016.

9. Brasil. Ministério da Saúde. Secretaria de Atenção à Saúde. Departamento de Atenção Básica. Política de Alimentação e Nutrição. Brasília: MS; 2012.

10. Brasil. Ministério da Saúde. Plano de Ações Estratégicas para o Enfrentamento das Doenças Crônicas não Transmissíveis (DCNT) no Brasil 2011-2022. Brasília: MS; 2011.

11. Brasil. Ministério da Saúde. Obesidade. Cadernos de Atenção Básica, n. 12. Série A. Normas e Manuais Técnicos. Brasília: MS; 2006.

12. Brasil. Ministério da Saúde. Portaria n 424, de 19 de março de 2013. Redefine as diretrizes para a organização da prevenção e do tratamento do sobrepeso e obesidade como linha de cuidado prioritária da Rede de Atenção à Saúde das Pessoas com Doenças Crônicas. Diário Oficial da União 2013; 28 jun.

13. Jaime PC, Silva ACF, Lima AMC, Bortolino GA. Ações de alimentação e nutrição na atenção básica: a experiência de organização no Governo Brasileiro. Cad. Saúde Pública. $2011 ; 24: 809-824$.

14. Vasconcelos ACCP, Magalhães R. Práticas educativas em Segurança Alimentar e Nutricional: reflexões a partir da experiência da Estratégia Saúde da Família em João Pessoa, PB, Brasil. Interface: Comunicação, Saúde, Educação. 2016; 20(56):99-110.

15. Cervato-Mancuso AM, Fiore EG, Redolfi SCS. Educação e Educação alimentar e nutricional. In: Cervato-Mancuso AM, Fiore EG, Redolfi SCS, organizadores. Guia de Segurança Alimentar e Nutricional. São Paulo: Manole; 2015. p.39-55.

16. Instituto Brasileiro de Geografia e Estatística - IBGE. Censo Demográfico 2000-2010: Agregado por Setores Censitários dos Resultados do Universo. Rio de Janeiro; 2010. [atualizado em 28 jul. 2016; acesso em 28 jul 2016]. Disponível em: http://www.ibge.gov.br/

17. Instituto Brasileiro de Geografia e Estatística - IBGE. Pesquisa Nacional por Amostra de Domicílios. PNAD Segurança Alimentar 2009. Rio de Janeiro; 2010. [atualizado em 28 jul. 2016; acesso em 28 jul 2016]. Disponível em: http://www.ibge.gov.br/

18. Bogdan R, Biklen S. Investigação Qualitativa em Educação:Uma Introdução à Teoria e aos Métodos. In: Bogdan R, Biklen S, oragnizadores. Investigação Qualitativa Aplicada em Educação: avaliação, pedagogia e ação. Porto: Porto Editora; 1994. p. 261-302.

19. Canesqui AM, Diez-Garcia RW. Antropologia e nutrição: um diálogo possível. In: Rio de Janeiro: Editora FIOCRUZ; 2005. 
ISSN 2179-6750

20. Brasil. Lei Orgânica de Segurança Alimentar e Nutricional, $n^{\circ} 11.346$, de 15 de setembro de 2006. Cria o Sistema Nacional de Segurança Alimentar e Nutricional - SISAN com vistas em assegurar o direito humano à alimentação adequada e dá outras providências. Diário Oficial da União 2006; 18 set.

21. Lopes RE, Borba PLO, Trajber NKA, Silva CR, Cuel BT. Oficinas de atividades com jovens da escola pública: tecnologias sociais entre educação e terapia ocupacional. Interface: Comunicação, Saúde, Educação. 2011; 15(36):277-288.

22. Maciel ALS, Fernandes RMC. Tecnologias sociais: interface com as políticas públicas e o Serviço Social. Serv. Soc. 2011; 105:146-165.

23. Castro IRR, Castro LMC, Gugelmim SA. Ações educativas, programas e políticas envolvidos nas mudanças alimentares. In: Diez-Garcia $\mathrm{R}$, Cervato-Mancuso A, oragnizadores. Mudanças alimentares e educação nutricional. Rio de Janeiro: Guanabara Koogan; 2011. p. 19-34.

24. Brasil. Ministério da Saúde. Secretaria de Gestão Estratégica e Participativa. II Caderno de educação popular em saúde. Brasília: MS; 2014.

25. Ferreira Neto JL, Kind L. Práticas grupais em Saúde. In: Promoção da Saúde: práticas grupais na estratégia Saúde da Família. São Paulo: Hucitec e Belo Horizonte: Fapemig; 2011. p. 55-84.

26. Botelho FC, Guerra LDS, Pava-Cárdenas A, Cervato-Mancuso AM. Estratégias pedagógicas em grupos com o tema alimentação e nutrição: os bastidores do processo de escolha. Ciênc Saúde Colet. 2016; 21(6):1889-1898.

27. Conselho Nacional de Segurança Alimentar e Nutricional - CONSEA. Relatório Final III Conferência Nacional de SAN: por um desenvolvimento sustentável com soberania e segurança alimentar e nutricional. Fortaleza; 2007. [atualizado em 28 jul. 2016; acesso em 28 jul 2016]. Disponível em: http://www4.planalto.gov.br/consea/publicacoes

28. Teo CRPA, Ferraz L, Cembranel F. Agentes Comunitários de Saúde: percepções sobre alimentação no contexto da Estratégia Saúde da Família. Ciência, Cuidado e Saúde. 2015; 14(3):1229-1236.

29. Oliveira e Silva D, Recine EGIG, Queiroz EFO. Concepções de profissionais de saúde da atenção básica sobre a alimentação saudável no Distrito Federal, Brasil. Cad. Saúde Pública. 2002; 18(5):1367-1377.

30. Rigo N, Cobayashi F. Oficinas culinárias para promoção da alimentação saudável: uma experiência na Amazônia Ocidental Brasileira. In: Cardoso MA, organizador. Nutrição em 262 
ISSN 2179-6750

Saúde Coletiva. São Paulo: Atheneu; 2014. p. 315-320.

31. Kinupp VF, Lorenzi H. Plantas alimentícias não convencionais (PANC) no Brasil: guia de identificação, aspectos nutricionais e receitas ilustradas. Nova Odessa: Plantarum; 2014.

32. Brasil. Ministério da Agricultura, Pecuária e Abastecimento. Secretaria de Desenvolvimento Agropecuário e Cooperativismo. Hortaliças não converncionais: (tradicionais). Brasília: MAPA; 2010.

33. Polain JP, Proença RPC. O espaço social alimentar: um instrumento para o estudo dos modelos alimentares. Rev. Nutr. 2003; 16(3):245-256.

34. Jaime PC, Santos LMP. Transição nutricional e a organização do cuidado em alimentação e nutrição na Atenção Básica em Saúde. Revista Divulgação em Saúde para Debate. 2014; $51: 72-85$.

35. Malta DC, Gosch CS, Buss P, Rocha DG, Rezende R, Freitas PC, Akerman M. Doenças Crônicas Não Transmissíveis e o suporte das ações intersetoriais no seu enfrentamento. Ciên Saúde Colet. 2014; 19(11):4341-4350.

36. Pinheiro R, Silva Junior AG. A centralidade do usuário na avaliação em saúde: outras abordagens. In: Pinheiro R, Martins PH, organizadores. Avaliação em saúde na perspectiva do usuário: abordagem multicêntrica. Rio de Janeiro: Cepesc; 2009. p. 37-52.

37. Lefevre F, Lefevre AMC. Health, empowerment and triangulation. Saúde e Sociedade. 2004; 13(2):32-38 